\title{
¿Puede ser el policosanol un arma contra la COVID-19?
}

\author{
Can policosanol be a weapon against COVID-19? \\ O policosanol pode ser uma arma contra o COVID-19?
}

Carlos Enrique SalgadoFuentes ORCID: 0000-0002-5918-0793

Universidad de Ciencas Médicas de Guantánamo. Guantánamo.

Emmanuel Zayas Fundora ORCID: 0000-0002-3830-358X

Hospital Clínico Universitario Manuel "Piti" Fajardo. Universidad de

Ciencias Médicas de La Habana. Facultad de Ciencias Médicas "Manuel Fajardo". La Habana.
La pandemia de la COVID-19 ha alcanzado un acelerado crecimiento a nivel mundial afectando de forma importante los sistemas sanitarios de más de 190 países. Aunque la insuficiencia respiratoria continúa siendo la característica distintiva y la principal causa de muerte en los casos de COVID-19 de gravedad moderada o alta, se han descrito varias complicaciones cardiovasculares y numerosos casos de enfermedad tromboembólica. La enfermedad grave por SARS-CoV-2 se asocia a alteraciones evidentes de la hemostasia representadas principalmente por fenómenos trombóticos que pueden presentarse en todos los lechos vasculares. ${ }^{(1,2)}$

En el Hospital Universitario 12 de Octubre durante los meses de marzo y abril de 2020 (el pico más alto de infección en Madrid) fueron atendidos 2.943 pacientes confirmados con COVID-19. De ellos, 106 pacientes presentaron procesos trombóticos vasculares (arteriales o venosos) sintomáticos; 11 pacientes presentaron trombosis vasculares múltiples en diferentes territorios y de diferente naturaleza. Martínez Chamorro y col. concluyeron que los pacientes con COVID-19 tienen una prevalencia aumentada de tromboembolismo pulmonar (26\%). Estos resultados demuestran un vacío importante en cuanto a las conductas terapéuticas de estos pacientes y la necesidad de buscar alternativas terapéuticas. ${ }^{(3,4)}$

Los mecanismos fisiopatológicos involucrados en la coagulopatía en COVID-19 no se conocen completamente, sin embargo, existen evidencias que ayudan a explicarla. Un punto fundamental en la fisiopatología es la endotelitis, caracterizada por disfunción, lisis y muerte endotelial, lo cual a su vez genera exposición de las capas que se encuentran por debajo del endotelio, denominado subendotelio, que a diferencia del endotelio es una superficie trombogénica a la cual se adhieren las plaquetas tapizando toda el área que quedó expuesta. También se induce un estado protrombótico debido a la libreación de mediadores de inflamación entre los que se destaca el Tromboxano A2..$^{(2-5)}$

Los fenómenos mencionados ocasionan un estado de trombofilia, que en buena parte es producto de la hiperagregabilidad plaquetaria y además por la liberación de vesículas o micropartículas, dando de esta manera un importante lugar a los antiagregantes plaquetarios en el plan terapéutico para la COVID-19.

Entre estos fármacos se destacan los salicilatos (AAS), los cuales suprimen la función plaquetaria en gran parte mediante la inhibición inducida por acetilación irreversible de la serina 530 de la ciclooxigenasa-1 (COX-1) y, en consecuencia, se inhibe la síntesis del tromboxano A2 (TXA2), el cual es producido por las plaquetas activadas. Sin embargo múltiples fármacos que han demostrado importantes efectos antiagregantes no han sido valorados actualmente para su administración en pacientes COVID-19 positivos. 
El Policosanol es uno de esos fármacos que podrían tener un efecto positivo en el pronóstico y evolución de los pacientes. ${ }^{(5)}$

El policosanol, mezcla de 8 alcoholes alifáticos primarios de alto peso molecular purificada de la cera de caña, produce efectos pleiotrópicos, entre los que se destaca su acción antiagregante plaquetaria demostrada experimental y clínicamente, la cual se acompaña de una reducción de las cifras plasmáticas de TXA2 y una tendencia al aumento de PGI 2.

Se ha demostrado que el policosanol inhibe la actividad de la COX-1, lo que podría constituir el fundamento de su acción antiagregante. Además, estudios experimentales y clínicos han demostrado que el policosanol produce efectos antioxidantes, y reduce las células endoteliales circulantes en plasma. ${ }^{(6)}$

Valdes y col. demostraron que dosis únicas de este medicamento (10-50 mg) inhibieron significativa y modestamente $(<20 \%)$ la agregación plaquetaria a la epinefrina y al adenosín difosfato (ADP) en voluntarios sanos, mientras $20 \mathrm{mg} /$ día durante 7 días redujo la agregación por epinefrina (22,5\%), ADP $(21 \%)$ y colágeno $(11,6 \%)$. Una dosis menor (10 mg/día) durante más tiempo (14 días) inhibió la agregación por Ácido araquidónico (25,8\%), epinefrina (17,8\%), y colágeno $(16,0 \%) .{ }^{(7)}$

Arruzazabala y col. Identificaron que el policosanol $(20 \mathrm{mg} / \mathrm{día})$ administrado por 7 días fue más efectivo en inhibir la agregación a la epinefrina $(32,6 \%)$ y al ADP $(37,3 \%)$ que el AAS $(100 \mathrm{mg} /$ día), el cual inhibió más la agregación al colágeno $(61,4 \%)$, inhibió la agregación por epinefrina $(21,9 \%)$ pero no al ADP. La terapia policosanol + AAS redujo marcadamente la agregación al colágeno $(71,3 \%)$, epinefrina $(57,5 \%)$ y ADP $(31,0 \%){ }^{(8)}$

Teniendo en cuenta lo planteado anteriormente sería adecuado valorar el potencial del policosanol como fármaco coadyuvante en el tratamiento de los pacientes COVID-19 positivos desde etapas iniciales de la enfermedad, teniendo esta intervención objetivos preventivos con respecto a las complicaciones trombóticas. Sería oportuna la realización de estudios rigurosos enfocados a la comprobación objetiva de estas posibilidades terapéuticas.

\section{Conflicto de interés}

Los autores no recibieron financiación para el desarrollo de la presente investigación.

\section{Bibliografía}

1- Rey JR, Caro-Codón J, Poveda Pineda D, Merino JL, Iniesta ÁM, López-Sendón JL. Complicaciones arteriales trombóticas en pacientes hospitalizados con COVID-19. Rev Esp Cardiol. 2020; 73(9), 769771. https://doi.org/10.1016/j.recesp.2020.05.013

2- González-Villalva A, Peña-Díaz A de la, Rojas-Lemus M, López-Valdez N, Ustarroz-Cano M, GarcíaPeláez I, Bizarro-Nevares P, Fortoul TI. Physiology of Hemostasis and its Alteration by Coagulopathy in COVID-19. Rev Fac Med UNAM. [Internet] 2020 [citado 17/07/2021];63(5), 45-57. Disponible en: https://www.medigraphic.com/cgi-bin/new/resumenl.cgi?IDARTICULO=95818

3- Gonzalez-Fajardo JA, Ansuategui M, Romero C, Comanges A, Gómez-Arbeláez D, Ibarra G, Garcia-Gutierrez A. Mortalidad de los pacientes covid-19 con complicaciones trombóticas. Med Clín. 2020; 156(3), 112-117. https://doi.org/10.1016/j.medcli.2020.10.004

4- Martínez Chamorro E, Revilla Ostolaza TY, Pérez Núñez M, Borruel Nacenta S, Cruz-Conde Rodríguez-Guerra C, Ibáñez Sanz L. Tromboembolismo pulmonar en pacientes con COVID-19: Estudio de prevalencia en un hospital terciario. Radiol. 2021; 63(1), 13-21. https://doi.org/10.1016/j. rx.2020.09.010

5- Aguirre Chang G, Trujillo Figueredo A. COVID-19: una enfermedad viral trombogénica por hiperactividad plaquetaria, liberación de vesículas extracelulares, nets y otros factores. ResearchGate [Internet] 2020 [citado 17/07/2021]. Disponible en: https://www.researchgate. net/publication/346048934_COVID-19_UNA_ENFERMEDAD_VIRAL_TROMBOGENICA_POR HIPERACTIVIDAD_PLAQUETARIA_LIBERACION_DE_VESICULAS_EXTRACELULARES_NETS_Y_ OTROS_FACTORES

6- Sánchez López JV. Efecto del policosanol en la recuperación del ictus isquémico (Doctor). La Habana: Instituto de Neurología y Neurocirugía. [Internet] 2014 [citado 17/07/2021]. Disponible en: http://tesis.sld.cu/FileStorage/000008-63BE-Javier_S\%C3\%A1nchez.pdf

7- Arruzazabala ML, Valdés S, Más R, Carbajal D, Fernández L. Comparative study of policosanol, aspirin and the combination therapy policosanol-aspirin on platelet aggregation in healthy volunteers. Pharmacological Research. 1997; 36(4), 293-297. https://doi.org/10.1006/phrs.1997.0201 
8- Valdes S, Arruzazabala ML, Fernandez L, Más R, Carbajal D, Aleman C, Molina V. Effect of policosanol on platelet aggregation in healthy volunteers. International Journal of Clinical Pharmacology Research. [Internet] 1996 [citado 17/07/2021];16(2-3): 67-72. Disponible en: https://europepmc.org/article/ med/9063758

\section{Respuesta carta al editor}

Noelia Speranza Mourine ORCID: 0000-0002-3330-9974

Profesora Agregada Departamento

Farmacología y Terapéutica. Facultad de Medicina. UdelaR. Uruguay.

Steffano Fabbiani ORCID: 0000-0002-8720-7228 Asistente Departamento de

Farmacología y Terapéutica. Facultad de Medicina. UdelaR. Uruguay.
La infección por SARS-CoV-2, denominada COVID-19, induce un estado protrombótico, con generación de trombos y fibrina que se deposita en la microcirculación, caracterizado por la elevación del fibrinógeno y dímeros-D. La hipoxemia grave secundaria a infección por SARS-CoV-2 se debe fundamentalmente a microtrombosis difusa y disfunción endotelial de la circulación pulmonar. ${ }^{1}$

Por lo tanto, la COVID-19 predispone a una incidencia aumentada de eventos trombóticos agudos, arteriales y venosos. De tal modo, múltiples consensos publicados proponen la tromboprofilaxis con heparinas de bajo peso molecular (HBPM) como primera opción, en ausencia de contraindicaciones, en todo paciente que requiere ingreso hospitalario por COVID-19, si bien para la Organización Mundial de la Salud (OMS) es una recomendación condicional con bajo nivel de evidencia. La anticoagulación debería reservarse para los diagnósticos confirmados de enfermedad tromboembólica venosa. ${ }^{2}$

El policosanol, cuyo código ATC es $\mathrm{C} 10 \mathrm{AX} 08^{3}$, es una mezcla de alcoholes alifáticos aislado de la caña de azúcar (Saccharum officinarum, L.), que puede considerarse un fitomedicamento, entendiendo como tal a cualquier medicamento de origen vegetal elaborado con extractos estandarizados, formados por partes aéreas o subterráneas de plantas u otro material vegetal. ${ }^{4,5}$

Policosanol inhibe la síntesis de colesterol en una etapa anterior a la formación del mevalonato, mediante la regulación de la HMGCoA reductasa. ${ }^{6}$

Se ha demostrado que reduciría la agregación plaquetaria inducida con ácido araquidónico, colágeno, adrenalina y difosfato de adenosina (ADP) en voluntarios sanos y en pacientes con hipercolesterolemia o diabetes tipo 2, efecto relacionado con la reducción de los niveles séricos de tromboxano A2 y aumento de prostaciclina. No afecta la coagulación. ${ }^{6}$

Es un fitomedicamento de origen cubano, donde se encuentra aprobado, además de República Dominicana, entre otros países de latinoamérica, y en Canadá como un producto natural. Está aprobado en Cuba en pacientes con hipercolesterolemia tipo lla y tipo Ilb, cuando la dieta sola sea insuficiente para controlar los niveles séricos de colesterol total y de lipoproteínas de baja densidad (LDL). Además está indicado como antiagregante plaquetario. ${ }^{7}$ No se encuentra registrado ni comercializado en Uruguay.

Considerando que el uso de fitomedicamentos con frecuencia es una práctica con escasa evidencia en cuanto a eficacia, efectividad y seguridad, es necesario previo a considerarlo una opción terapéutica evaluarlo con fármacos de referencia que ya han demostrado efecto antiagregante plaquetario, a destacar el ácido acetilsalicílico (AAS), por su similar mecanismo de acción antiagregante plaquetario.

Como con cualquier otro principio activo con potencial efecto terapéutico sería interesante conocer su rol en la clínica para el mismo. Sin embargo, no hay estudios clínicos en curso, por lo menos en la base de datos de estudios clínicos de la National Institute of Health (NIH) de Estados Unidos que evalúen la eficacia de este medicamento como antitrombótico. ${ }^{8}$

En caso que se utilice como antiagregante plaquetario en medios donde no se encuentra registrado o fuera de las indicaciones aprobadas o menos documentadas, debería ser bajo una estricta monitorización, idealmente en el contexto de un estudio clínico. Como establece la OMS, puede ser éticamente apropiado ofrecer a los pacientes intervenciones experimentales de emergencia fuera de los ensayos clínicos, siempre que no exista un tratamiento eficaz comprobado; no es posible iniciar estudios clínicos de inmediato; el paciente ha dado su consentimiento informado; y se supervisa el uso de emergencia de la intervención, y los resultados se documentan y se comparten de manera oportuna con la comunidad médica y científica en general. ${ }^{9}$ 


\section{Bibliografía}

1- López-Reyes R, Oscullo G, Jiménez D, Cano I, García-Ortega A. Riesgo trombótico y COVID-19: revisión de la evidencia actual para una mejor aproximación diagnóstica y terapéutica. Arch Bronconeumol. 2021;57(S1):55-64.

2- Organización Mundial de la Salud. Guía viva del manejo de la COVID-19 de la OMS [Internet] Ginebra: OMS, 2021 [citado 01/010/2021]. Disponible: https://www.who.int/publications/i/item/WHO-2019nCoV-clinical-2021-1

3- Organización Mundial de la Salud. Centro colaborador para la metodología de las estadísticas de drogas. Policosanol. [Internet] Ginebra: OMS, 2021 [citado 01/10/2021] Disponible en: https://www. whocc.no/atc_ddd_index/?code=C10AX08

4- Morales MA, Morales JP. Plantas medicinales, fitofarmacos y fitomedicamentos: hacia una fitomedicina basada en la evidencia científica. Plantas Medficinales y Medicina Natural Biomedicina. 2012; 7(3): $41-54$

5- Moreale J, González T. Evaluación de las interacciones farmacológicas de las hierbas medicinales en Uruguay. Biomedicina. 2012; 7 (3):6-21.

6- Centro para el Control Estatal de Medicamentos, Equipos y Dispositivos médicos Ficha técnica Ateromixol [Internet]. La Habana: CECMED, 2020 [citado 01/10/2021]. Disponible en: https://www. cecmed.cu/registro/rcp/medicamentos/ateromixolr-20

7- Cuba. Ministerio de Salud Pública. Formulario Nacional de Medicamentos de Cuba[Internet] La Habana: MSP, 2014[citado 01/10/2021]. Disponible en: http://fnmedicamentos.sld.cu/index. php?P=FullRecord\&ID=585

8- National Institute of health. Base de datos de estudios clínicos de Estados Unidos. Policosanol [Internet] NIH, 2021[citado 01/10/2021]. Disponible en: https://clinicaltrials.gov/ct2/ results? cond=policosanol\&term $=\&$ cntry $=\&$ state $=\&$ city $=\& d i s t=$

9- Organización Mundial de la Salud. Uso off-label para COVID-19[Internet]Ginebra: OMS, 2021[ citado 01/10/2021] Disponible en: https://www.who.int/news-room/commentaries/detail/off-label-use-ofmedicines-for-covid-19 